\title{
La Anomia como Moderador de la Relación entre Percepciones de Justicia Organizativa y el Uso Negligente de Internet en el Trabajo
}

\section{Anomia as a Moderator of the Relationship Between Perceptions of Organizational Justice and Cyberloafing}

\author{
Pablo Zoghbi Manrique de Lara \\ Universidad de Las Palmas de Gran Canaria
}

\begin{abstract}
Resumen. Este estudio analiza el papel moderador de la anomia en la relación entre las percepciones de justicia organizativa y el uso negligente de Internet por el empleado, o 'ciberpereza.' El modelo propuesto sugiere que la anomia interactúa con la justicia organizativa y la ciberpereza. Los datos fueron extraídos de una muestra de 270 (17.46\%) profesores de una universidad española, usando el correo electrónico vía Intranet. Regresiones Múltiples Jerárquicas muestran que la anomia actúa como moderador del vínculo justicia-ciberpereza, Este papel moderador resultó más acusado en el caso de la justicia procedimental. Los hallazgos contribuyen a un mejor conocimiento de cómo las percepciones de injusticia conducen al empleado a la ciberpereza. Finalmente se presentan las implicaciones, limitaciones y otras recomendaciones que del presente estudio parecen derivarse.
\end{abstract}

Palabras clave: ciberpereza, justicia organizativa, anomia, conductas contraproductivas.

\begin{abstract}
This article analyzes the moderating role of anomia in the relationship between perceptions of organizational justice and the employee misuse of Internet or cyberloafing. The model suggests that anomia interacts on the link between organizational justice and cyberloafing. Data were collected from a sample of $270(17.46 \%)$ teachers of a Spanish university, using e-mail via Intranet. Hierarchical multiple regression results show that anomia indeed acts a moderator of the link justice-cyberloafing. This moderating role was more relevant in the case of procedural justice. The findings contribute to a better knowledge of how injustice perceptions lead employees to cyberloafing. Finally, the implications and limitations of the study are presented as well as some recommendations.
\end{abstract}

Key words: cyberloafing, organizational justice, anomia, workplace deviance.

Durante la pasada década, el espectacular crecimiento del uso de Internet en las organizaciones ha permitido mejoras sustanciales en sus procesos productivos (p.e., utilización del e-servicio, creación de grupos virtuales, potenciación del teletrabajo). Desafortunadamente, también ha propiciado la aparición de un nuevo tipo de comportamiento contraproductivo: los empleados navegan por Internet cuando, durante sus jornadas de trabajo, deberían estar trabajando. A este uso fraudulento de Internet la literatura anglosajona le denomina 'cyber-loafing', o ciber-pereza. Probablemente, el uso inicial de la ciberpereza parte del artículo de Kamins (1995) titulado: Cyberloafing: Does Employee Time Online Add Up to Net Losses? [Ciberpereza: ¿Se contabiliza como pérdida el

Una versión abreviada de este trabajo ha sido publicada en la revista CyberPsychology \& Behavior como Rapid Communication. La correspondencia sobre este artículo deberá enviarse al autor a la siguiente dirección: Departamento de Economía y Dirección de Empresas, Área de Recursos Humanos, Universidad de Las Palmas de Gran Canaria, Campus Universitario Tafira, Edificio Empresariales, Módulo C-2.18, 35017 Las Palmas de Gran Canaria. E-mail: pzoghbi@dede.ulpgc.es tiempo del empleado conectado a Internet?], si bien su consagración académica es atribuible Vivien Lim (2002).

La ciberpereza se encuadra dentro de las conductas contraproductivas. Éstas se apartan de las exigidas contractualmente en el puesto de trabajo, en una dirección negativa y, en la literatura anglosajona, son denominadas genéricamente como workplace deviance. Robinson y Bennett las definen como aquellas a través de las cuales los trabajadores, "de forma voluntaria, violan importantes normas organizativas... poniendo en peligro el bienestar de la organización, de sus miembros, o de ambos a la vez" (1995, p. 556). En esta línea, Lim define la ciberpereza como: “...cualquier acto voluntario del empleado que suponga un uso del acceso a Internet de su organización, durante horas de oficina, para navegar por sitios 'Web' de interés personal—no profesional—, y para abrir su buzón de correo electrónico, tanto para recibir como enviar mensajes privados (Lim, 2002, p. 677)." En su estudio, Lim examinó una selección de empleados ciberperezosos confesos y descubre que la razón del uso fraudulento de Internet estriba más en una reacción hacia la percep- 
ción de un trato injusto por parte de su organización, que en los recurridos tópicos del empleado aburrido o perezoso que mata su tiempo conectado a Internet.

La literatura popular advierte del negativo impacto de la ciberpereza. Un estudio de 1.000 trabajadores en EE.UU. reveló que el 64\% de ellos navegaba habitualmente por Internet, para asuntos personales, durante sus jornadas de trabajo (The Straits Times, 2000). De acuerdo a esta literatura, el coste de la ciberpereza en términos de caída de la productividad del empleado puede estimarse entre el 30\% y el $40 \%$ (Verton, 2000), y costó a las organizaciones estadounidenses 5.300 millones de dólares en 1999 (Bronikowski, 2000). En el Reino Unido, según un estudio realizado por la firma 'Peninsula' (Management Issues News, 2004) sobre 3.245 trabajadores de 750 empresas, durante 2003 los empleados consumieron el $40 \%$ de su jornada diaria en la práctica de la ciberpereza. En España, este uso inapropiado de Internet ha llevado al Ministerio de Defensa a restringir el acceso al personal militar debido a reiterados colapsos sufridos en la red (EcoDiario, 2008).

La relación entre las percepciones de justicia organizativa y la ciberpereza recibió un importante apoyo en el estudio de Lim (2002). Sin embargo, esta relación está frecuentemente controlada o mediada con éxito por otras variables individuales o del contexto organizativo (Aryee et al., 2002). Así, Lim (2002) propuso 'La metáfora del Libro Mayor', un constructo desarrollado a través de la teoría sobre las 'técnicas de neutralización' (Robinson y Kraatz, 1998; Sykes y Matza, 1957). Según ésta, el empleado que percibe un trato injusto por parte de su organización tiende al victimismo, 'neutralizando' así su culpa, y ‘justificando' por tanto su ciberpereza arropado en la creencia de que 'su empresa se lo merece' - como si le amparase un saldo contable a su favor. También con respecto a la ciberpereza, Zoghbi (2006) encontró que la injusticia organizativa antecede a la ciberpereza, pero a través de la 'intimidación' que tal injusticia provoca en el empleado. Aryee et al., (2002) propusieron la 'confianza' también como variable mediadora. Consideraron que la justicia organizativa genera principalmente confianza e, indirectamente, a través de dicha confianza hacia la organización, conducta organizativa. También Moorman et al. (1998) estudiaron la mediación del 'apoyo organizacional'.

Figura 1. Modelo Propuesto que predice la Anomia como Variable Moderadora de la relación entre la Justicia Organizativa y Ciberpereza

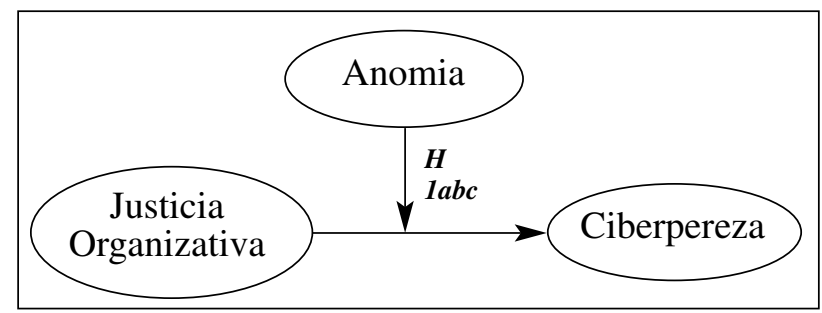

Revista de Psicología del Trabajo y de las Organizaciones Vol. 25, n. ${ }^{\circ}$ 2, 2009 - Págs. 99-112
Finalmente, Aquino et al. (2004:1002) afirmaron que “... no todo aquel que es tratado injustamente por su supervisor responde con conducta desviada." En su estudio, los citados autores propusieron variables moderadoras como la categoría laboral, los rasgos personales de agresividad, entre otros, como elementos que interactúan moderando la citada relación.

Aunque su primera concepción parte de la sociología, la anomia (del Griego, a-: ausencia, y -nomos: ley) pudiera también reflejar el desmoronamiento normativo-valorativo de un individuo en su trabajo: éste siente que su organización no le provee del contexto adecuado para que pueda cifrar su confianza sobre lo que legítimamente puede esperar de los demás y de su organización, en el transcurso de su interacción social. Además, desde esta perspectiva, la anomia parece presentarse como un fenómeno de interacción potencial con las percepciones de justicia organizativa y la ciberpereza. Tal aserto parece estar en consonancia con el hecho de que la anomia precisamente nace, a nivel sociológico, como un intento de explicar conductas desviadas en la sociedad y, por tanto, dentro de las organizaciones podría también propiciar conductas desviadas como la ciberpereza.

Sobre la base la literatura descrita anteriormente, el presente estudio propone el uso de la anomia como variable moderadora de la citada relación prediciendo que entre los empleados comparativamente más anómicos la relación injusticia-ciberpereza será más intensa. En la Figura 1 vemos como, dado que la literatura contempla tres tipos de justicia organizativa, esta moderación se concreta a través de tres sub-hipótesis (H1a, H1b, y H1c). Hasta donde sabemos, el uso de la anomia como moderador de esta relación no tiene precedentes en la literatura sobre Psicología del Trabajo y Comportamiento Organizacional.

\section{Antecedentes Teóricos}

Los teóricos del intercambio sugieren que tras las interacciones humanas subyace un fundamento económico, desde el momento en los sujetos sopesan los beneficios que obtienen de sus acciones hacia los demás (p.ej., Blau, 1964; Homans, 1961; Thibaut y Kelly, 1959). Así, Blau (1964) muestra como el individuo, en el marco del intercambio social, guía su comportamiento hacia aquellas acciones que le van a reportar recompensas, tratando de asegurar una "útil" dinámica de intercambios. Cuando ésta no se produce, sus respuestas cesan. Las relaciones entre la organización y sus miembros constituyen un marco habitual de tal intercambio. Desde tal contexto, cuando una de las partes (p.ej., el empleado) considera que la otra (p.ej., organización) traiciona este acuerdo tácito establecido, puede actuar en consecuencia y movido, por ejemplo, por la venganza o por su deseos de restablecer la equidad, pudiera incluso dañar a la otra parte. 
La literatura sobre las relaciones en el trabajo, cada vez más prolíficamente, ha ampliado el abanico de compensaciones que, más allá de un salario justo, los trabajadores esperan recibir de un estricto cumplimiento de sus obligaciones laborales. Desde el momento en que el trabajador puede valorar sus recompensas (derivadas de su aportación a la organización) como justas y equitativas o no, emerge el concepto de 'justicia organizativa.' Investigaciones previas la han identificado como multidimensional: (a) una justicia compensatoria o distributiva (JD), esto es, de recompensas justas, (b) una justicia de procedimiento (JP), o relativa a la mayor o menor justicia percibida de los procedimientos y rutinas de trabajo; $y$, (c) una justicia interpersonal (JI), o percibida según el trato amable y equitativo recibido por parte de los superiores (Folger y Cropanzano, 1998).

Dado que la política de compensaciones (p.ej., salarios y los ascensos) permiten al empleado cubrir un amplio abanico de necesidades, parece razonable pensar su especial sensibilidad a la injusticia distributiva (JD) (véase, p.ej., Analoui y Kakabadse, 1991). Pero también, dado que el uso del ordenador e Internet comprende actividades o tareas considerablemente estructuradas, en donde existen claras rutinas y procedimientos, también parece razonable afirmar que los trabajadores 'internautas' sean igualmente sensibles a la JP. Por último, dado el creciente incremento de los aspectos interpersonales en el contexto laboral, también la JI pudiera resultar importante en la configuración global de las percepciones de justicia organizativa de los trabajadores (p.ej., Skarlicki y Folger, 1997; Skarlicki et al., 1999).

La injusticia organizativa es frecuentemente citada como causa de conductas contraproductivas (DiBattista, 1989, 1996; Lim, 2002; Neuman y Baron, 1998; Robinson y Bennett, 1997; Skarlicki y Folger, 1997; Sieh, 1987; Tucker, 1993). Efectivamente, en la literatura se encuentran evidencias empíricas que sugieren que los empleados son más propensos a presentar conductas negativas cuando perciben un tratamiento injusto por parte de la organización. Por ejemplo, Greenberg (1990) encontró que los trabajadores que sufrían recortes salariales percibidos como injustos reaccionaban mediante robos; Skarlicki y Folger (1997) descubrieron que los empleados que sentían un trato injusto de la organización presentaban conductas vengativas contra ésta; Adams (1965) comprobó cómo una falta de equidad conduce al empleado a reducir sus esfuerzos de trabajo; y Aquino et al. (1999) descubrieron que la injusticia percibida en el trabajo se mostraba como causa central en la aparición de conductas desviadas. Por tanto, a la luz de la literatura sobre las teorías de intercambio social y de justicia organizativa, así como de conducta desviada, parece razonable augurar que si los individuos perciben un trato injusto (en cualquiera de sus tres facetas) por parte de la organización, tal circunstancia les provoque reacciones hacia su organización, les incite a una respuesta, y que ésta sea, lógicamente, 'contra' la organización percibida como fuente de dicha injusticia: su universidad.

\section{La Anomia Sociológica y la Anomia en las Organizaciones}

En dos obras clásicas, De la Division du Travail Social (1893) y Le Suicide (1897), Emile Durkheim desarrolla el concepto de anomia (del Griego, a-: ausencia, y -nomos: ley) como una patología social donde las normas bien no existen, se muestran caducas o confusas, o han perdido los valores que las sustentaban. Tal situación parece conducir una ausencia de un referente social sólido que propicia el desarraigo social del individuo en sus grupos sociales y en la sociedad misma, exponiéndole a conductas desviadas (Marks, 1974). Para Robert Merton (1957), desde la literatura sociológica americana, la anomia es producto del desajuste entre las metas culturales que la sociedad ofrece y la escasez de los medios que la misma sociedad proporciona para alcanzarlos. Por último, Bass (1990, p. 915) entiende la anomia como: “...la pérdida de control social contra la conducta desviada, debido a una disolución de las normas y estándares."

Para Merton (1957), la anomia tiene un claro sesgo psicológico y en esa dirección anota una interesante cita de MacIver (1950, p. 86) donde afirma que: “...la anomia significa un estado de ánimo del individuo cuyas raíces morales se han roto, que ya no tiene normas sino únicamente impulsos desconectados, que no tiene ningún sentido de continuidad, de grupo, de obligación ... el individuo anómico se ha hecho espiritualmente estéril, responsable sólo ante sí mismo, y ante nadie más. Se ríe de los valores de otros individuos. Su única fe es la filosofía de la negación. Vive en la delgada frontera de la sensación entre ningún futuro y ningún pasado." La descripción de MacIver (1950), citada por Merton (1957), concluye sosteniendo que anomia es un estado de ánimo en el que está roto, o mortalmente debilitado, el sentido de cohesión social, principal resorte moral del individuo.

Esta reformulación de la anomia a nivel individual la apartó un tiempo de la investigación empírica, hasta que Leo Srole (1956) desarrolló su constructo. Este constructo y su escala original de medida de cinco ítems no hacen referencia a contextos anómicos, sino al grado en que un individuo 'experimenta' la anomia en contraposición a la 'eunomia' (del Griego, eu-: posesión, y -nomos: ley). Merton (1957) sostiene que cada uno de los cinco ítems del cuestionario de Srole refleja un matiz anómico singular: 1) la percepción de que los líderes de la comunidad son indiferentes a las necesidades de uno; 2) la percepción de que es poco lo que puede lograrse en la sociedad que no sea fundamentalmente imprevisible y carente de orden; 3) la percepción de que los objetivos de la vida se alejan en 
vez de lograr alcanzarse; 4) una sensación de futilidad, y 5) el convencimiento de que no se puede contar con los demás para obtener apoyo social y psicológico.

A pesar de que Srole (1956) abre las puertas con su escala original de cinco ítems (que en 1972 fue ampliada a nueve) a la investigación organizativa, la anomia goza de un discreto desarrollo teórico en este campo. En general, la literatura organizativa la ha asociado con organizaciones carentes de valores o de un tejido normativo sólido. Por ejemplo, Allport (1924), afirma que cuando una organización no expresa claramente sus valores, se convierte en anómica. Shedd y Bachrach (1991) advierten de este peligro en organizaciones no suficientemente cohesionadas o sin soportes internos, o cuando están inmersas en procesos de crecimiento, expansión y de mayor especialización. Para Hodson (1999) las organizaciones anómicas son aquéllas cuyos centros de trabajo adolecen de un mínimo de normas generales que los regulen.

Sin embargo, desde la perspectiva individual que ofrece el constructo de Srole (1956), el hablar de 'organización anómica' no parece tener mucho sentido. Si para Srole la anomia es una sensación de desarraigo social, no parece que una organización pueda estar desarraigada de sí misma. Las consideraciones del párrafo anterior, desde un enfoque macrosocial, quizá sólo permitan hablar de una organización anómica como aquella 'que propicia patologías anómicas individuales' entre sus miembros. Sin embargo, estos antecedentes macrosociales de la anomia en los empleados no es lo que mide el constructo de Srole, sino que más bien referencia a las sensaciones de anomia cuyas causas pudieran estar en esas, aludidas, 'organizaciones anómicas.' Tampoco la escala de Srole mide 'conductas anómicas'. Estas, probablemente, no son otra cosa que consecuencias de las citadas sensaciones anómicas (McClosky y Schaar, 1965).

Por último, conviene insistir en que el origen sociológico del concepto de la anomia, no debe impedirnos su utilización en las organizaciones. Al proponer Srole su constructo como una percepción subjetiva-felt anomia - la contraposición sociológica versus organizativa no debe afectarnos ya que los sentimientos anómicos del constructo de Srole son siempre los mismos, independientemente de los antecedentes que los genere (Hagedorn y Labovitz, 1968). Otra cuestión sería la distinta 'sensibilidad' de la escala de Srole a los contextos sociológicos en comparación con los contextos más específicamente organizacionales. Tal extremo exige una leve adaptación de la escala de Srole a nuestro estudio en el marco de las organizaciones.

\section{El papel moderador de la Anomia en la relación Justicia-Ciberpereza}

Se mencionó anteriormente que el empleado que percibe una organización como injusta genera respues- tas, más o menos intensas, y más o menos generalizadas, contra la organización. Esas respuestas pudieran muy bien concretarse en ciberpereza. Ahora bien, en el caso del trabajador anómico, que percibe con dificultad a su organización como normativamente firme, que presencia un ambiente organizacional sin valores sólidos, parece razonable pensar que se mostrará más sensible a la relación básica planteada en nuestro trabajo: justicia y ciberpereza. ¿No es la percepción de justicia organizativa uno de los valores más sólidos para muchos trabajadores? Por tanto, parece fundado pensar que una situación anómica pueda interactuar con la variable justicia organizativa e intensificar, neutralizar o invertir sus efectos sobre la ciberpereza. En efecto, no debemos olvidar que la literatura sobre anomia muestra una clara relación entre anomia y conductas disfuncionales. Potter (1989) acepta que la anomia contribuye a incrementar, en un trabajador, su inclinación a mostrar conductas desviadas y Cohen (1995) planteó un modelo que relaciona la anomia con valores sociales, estrategia de negocio, cultura organizativa y conducta desviada en las empresas. También, la anomia se ha utilizado empíricamente como predictor de rotación en las organizaciones (Taylor y Zimmerer, 1992): bajos y medios niveles de anomia en supervisores constituyeron una causa significativa de indeseables niveles de rotación. Por tanto, creemos que la literatura aporta indicios de su posible efecto interactivo con las percepciones justas o injustas de la organización y con las respuestas disfuncionales.

La triple respuesta actitudinal cognitiva, afectiva y conductual puede explicar el mecanismo a través del cual las percepciones de injusticia organizativa construyen actitudes negativas hacia la organización que se traducen, como hemos visto, en respuestas conductuales desviadas. Si la anomia refleja, en el foro interior del empleado, el desmoronamiento de normas y valores organizacionales, este contexto puede perfilarse como caldo de cultivo en el fortalecimiento de actitudes negativas contra la organización y, por ende, de conductas contra la misma. Apoyando esta idea, Hackman y Oldham (1976) vinculan actitudes desfavorables hacia los jefes/superiores con conductas negativas hacia sus tareas (¿ciberpereza?), subrayando la 'sensación de falta de apoyo' (¿anomia?) que catalizaría este proceso. En esta línea, parece razonable pensar que la JI puede reflejar alguna de dichas actitudes negativas hacia la jefatura. Por último, McClosky y Schaar (1965) muestran la influencia de la ausencia del apoyo del jefe sobre la anomia de los trabajadores, lo que podría extrapolarse a la justicia organizativa dado que el apoyo del jefe parece un elemento estrechamente relacionado con la percepción de JI del empleado (véase, p.ej., Moorman, et al., 1998).

Por tanto, en base al marco teórico expuesto, y a los argumentos aportados creemos existen indicios fundados para sugerir las siguientes hipótesis (véase la Figura 1): 
Hipótesis 1a: Entre los individuos menos anómicos, comparados con los más anómicos, sus percepciones de Justicia Distributiva (JD) se relacionarán más negativamente con su ciberpereza.

Hipótesis 1b: Entre los individuos menos anómicos, comparados con los más anómicos, sus percepciones de Justicia Procedimental (JP) se relacionarán más negativamente con su ciberpereza.

Hipótesis 1c: Entre los individuos menos anómicos, comparados con los más anómicos, sus percepciones de Justicia Interpersonal (JI) se relacionarán más negativamente con su ciberpereza.

\section{Método}

\section{Muestra}

La muestra la constituyen $270(17.46 \%)$ de los 1.546 profesores de una universidad pública española. Del universo, en el transcurso de la investigación, 932 eran funcionarios de carrera $(60.28 \%)$ mientas que el resto, 615, disponían de otra vinculación legal. Por edades, 501 (el 32.5\%) tenían 40 años o menos, y sólo 89, el 5.7\%, más de 60 años. Por sexos, el $68 \%$ eran varones y el $32 \%$ eran mujeres. Se envió un cuestionario por correo electrónico con dos recordatorios, el 5 de octubre, y el 11 de noviembre de 2004, si bien las repuestas fluían a ritmo continuo y estable. Se instaló, para ello, un cuestionario en la intranet de la universidad, al que se facilitó el acceso a través de un enlace en la misma misiva electrónica en la que se solicitaba la colaboración. Previamente, el proyecto recibió aprobación oficial garantizándose, así, el anonimato. No hay constancia de diferencias en la accesibilidad de los profesores a su buzón individual de correo electrónico, ni de diferentes predisposiciones en las respuestas que pudieran afectar la aleatoriedad de la muestra. Las respuestas válidas fueron 270, tras rechazar 12 al ser cumplimentadas insuficientemente o por incoherencia en los datos. Por sexo, el $63.6 \%$ de la muestra son varones y el $35.4 \%$ mujeres. Por edades, el $40 \%$ tiene 40 años o menos y sólo el $4.2 \%$ tiene más de 60 años. Por categorías, el $68 \%$ es funcionario, el resto mantiene una vinculación no funcionarial. Como se puede apreciar, las estructuras descriptivas de la muestra y de la población son bastante similares.

\section{Medición de Variables}

Todos los ítems del estudio fueron registrados sobre una escala de 7 puntos, oscilando desde (1) Máximo desacuerdo a (7) Máximo acuerdo-en Ciberpereza de (1) Nunca a (7) Constantemente. A fin de aumentar la validez de las repuestas algunos ítems estaban invertidos en el cuestionario, lo que exigió su posterior recodificación. Todos los ítems utilizados en este estudio pueden verse en la Tabla 1. La fiabilidad fue establecida a través del alfa de Cronbach, cuyos valores aparecen en la diagonal principal de la tabla de correlaciones (Tabla 2).

Justicia Organizativa. Las tres variables de justicia fueron medidas usando las escalas desarrolladas por Moorman (1991). La justicia distributiva (JD) fue medida con cinco ítems $(\alpha=0.921)$ que intentaban recoger las percepciones de los individuos del grado en que se sienten justamente recompensados por su organización. La justicia procedimental (JP) fue calibrada mediante seis ítems $(\alpha=0.961)$ que buscaban registros de percepciones de equidad de los procedimientos organizacionales. La escala de justicia interpersonal (JI) incluyó seis ítems para valorar si los procedimientos organizacionales fueron aplicados con propiedad y justicia por los supervisores. El alfa de Cronbach de esta escala fue 0.949. Los resultados de un análisis factorial confirmatorio (AFC) se muestran en la Tabla $1 \mathrm{y}$ sugieren que las tres justicias son tres constructos distintos, en línea con la investigación previa de la literatura (p.ej., Moorman, 1991; Niehoff y Moorman, 1993).

Anomia Organizativa. La escala utilizada para medir esta variable está basada en la original de 5ítems de Srole (1959). Dado que la escala fue originalmente diseñada para recoger la 'anomia sentida' en la sociedad, pareció conveniente expandirla con tres ítems adicionales, de tal manera que aludiera más explícitamente a elementos del entorno organizacional universitario y, por tanto, 'recogiera' al máximo la anomia que pudiera provenir de dicho contexto. Los ítems añadidos fueron 'Me siento muy integrado en la universidad (R)', 'A veces no puedo dejar de preguntarme qué hago yo en un trabajo como éste', 'A veces pienso que no sé que es lo que la universidad realmente quiere de nosotros' (véase la Tabla 1) Dichos ítems fueron elaborados especialmente para el estudio, en línea con la literatura existente sobre la anomia. También revisamos otras escalas, como la de McClosky y Schaar (1965), pero sólo con la intención de mejorar el denominado 'back-translation' en el proceso de traducción. Así, finalmente dispusimos de una escala final de ocho ítems $(\alpha=0.839)$ para medir los desórdenes anómicos percibidos por el profesorado universitario.

Ciberpereza. Esta escala fue una versión más reducida de la propuesta por Lim (2002), la cual incluye ocho ítems referidos a curiosear por Internet y tres ítems más a consultar y usar el buzón electrónico privado (no profesional). Seleccionamos los más idóneos, para el contexto de estudio, cuatro del primer bloque y uno del segundo $(\alpha=0.862)$, con pretensiones de unidimensionalidad, que el AFC confirma.

Variables de control. Basándonos en la literatura, consideramos dos variables, sexo $(1=$ hombre, $2=$ mujer) y edad ( $1=$ de 23 a 30; $2=31$ a $40 ; 3=41$ a 50 ; $4=51$ a $60 ; 5=61$ a $70 ; 6=71$ a 75 ), las cuales pueden covariar con las demás variables dependientes e 
independientes del estudio $\mathrm{y}$, por tanto, podrían ser incluidas como variables de control en nuestros análisis (p.ej., Zellars et al., 2002; Aquino et al., 2004).

Sobre la base de la metodología SEM (Structural Equation Modeling) utilizada, procedimos a realizar un AFC con todas las variables del estudio para asegu- rarnos que son, efectivamente, constructos diferentes (Long, 1983; Jöreskog y Sörbom, 1979; Bollen, 1989). El ajuste total de las variables fue estimado usando varios índices: (1) Cmin = mínima discrepancia, test estadístico del ratio de verosimilitud $\chi^{2},(2) d f=$ grados de libertad, (3) $p=$ nivel de significación, (4) CFI

Tabla 1. Resultados del Análisis Factorial Confirmatorio (AFC) de todas las variables de este estudio

Ítems de las variables

Carga Factorial

Justicia Distributiva (JD) $(\alpha=0.921)$

Sientes que la universidad te ha estado recompensando justamente...

...por la formación y experiencia que aportas a la universidad

...por el trabajo bien hecho que realizas

...por el esfuerzo que pones en tu trabajo

0.88

...por las tensiones y presiones que soportas en tu trabajo

0.88

...por las responsabilidades que asumes

Justicia en los Procedimientos o Procedimental (JP) $(\alpha=0.961)$

Sientes que los procedimientos de la universidad han sido diseñados adecuadamente en orden a...

...escuchar la opinión de los implicados o afectados en las decisiones que la universidad toma

...incluir a todas las partes afectadas en el proceso de toma de decisiones

0.92

...permitir formular aclaraciones, o información adicional, sobre la decisión que toman

....ayudarte a informarte con precisión para que puedas opinar sobre las decisiones que va a tomar

0.90

...proporcionar oportunidades para discutir o recurrir un decisión tomada

...informarte sobre decisiones que la universidad toma, y su implantación, en materias que te afectan

Justicia en la interrelación con los supervisores $(J I)(\alpha=0.949)$

Sientes que en la universidad...

...tu jefe pone de su parte para conseguir tener contigo una relación profesional sincera y auténtica 0.92

...tu jefe toma en consideración tus puntos de vista

...tu jefe te explica oportunamente el porqué de las decisiones que te afectan

...tu jefe evita caer en prejuicios personales hacía ti

...tu jefe te trata con amabilidad y consideración

...tu jefe muestra interés sobre tus derechos como trabajador

Anomia $(\alpha=0.839)$

Tal y como están las cosas en la universidad es difícil ilusionarse con el futuro

A pesar de lo que nos quieran decir, en esta universidad, en general, la gente cada vez está peor 0.75

La mayoría de los empleados de esta universidad no se interesan por los problemas de los demás 0.67

A veces pienso que no sé que es lo que la universidad realmente quiere de nosotros 0.66

En mi trabajo no se sabe con quién uno puede contar de verdad 0.63

$\begin{array}{ll}\text { Me siento muy integrado en la universidad }(\mathrm{R}) & 0.50\end{array}$

A veces no puedo dejar de preguntarme qué hago yo en un trabajo como éste $\quad 0.49$

$\begin{array}{ll}\text { En mi trabajo hay que vivir el hoy, en el futuro prefiero no pensar } & 0.44\end{array}$

Ciberpereza $(\alpha=0.862)$

Me he pasado usando Internet en mi trabajo para...

...visitar portales o periódicos digitales para buscar información personal

0.80

...visitar alguna página para bajarme algún programa o fichero para uso personal o familiar 0.78

...enviar o abrir correos personales, no profesionales

0.78
0.75

...visitar la Web de mi cuenta corriente de mi banco o caja

0.74

...navegar y así evadirme un poco

0.65

Chi-cuadrado $=637.009 \mathrm{df}=395 \mathrm{p}=$ sig. $\mathrm{Cmin} / \mathrm{df}=1.613$

$\mathrm{CFI}=0.961 \mathrm{NFI}=0.905 \mathrm{RMSEA}=0.048 \mathrm{TLI}=0.954 \mathrm{PCFI}=0.816 \mathrm{PNFI}=0.769$

Nota: El ajuste es bueno si Chi-cuadrado p > 0.05; CFI, NFI, y TLI están en el intervalo (0.1), y mejor si se aproximan a 1; RMSEA $<0.05$. (R) Ítems registrados inversamente. 
= Índice de ajuste comparativo, (5) NFI = Índice de ajuste normalizado, (6) RMSEA = Raíz cuadrada del error promedio, (8) PCFI = ajuste CFI por el número de grados de libertad, (9) PNFI = Índice de ajuste normalizado de la parsimonia, (10) TLI = Indice de Tucker-Lewis.

Los resultados del AFC, mostrados en la Tabla 1 y en su estructura completa en el anexo, sugieren, como ya hemos comentado, que las cinco variables forman constructos que están relacionados pero que son distintos.

\section{Resultados}

La Tabla 2 muestra los estadísticos descriptivos, las correlaciones $(r)$ y las fiabilidades de todas las variables del estudio. Los resultados del análisis de las correlaciones parecen ya apuntar aspectos que en el marco teórico del estudio se auguraban. Por ejemplo, la anomia parece interactuar intensa y negativamente con las tres justicias organizativas $(r=-0.48 ; p<0,001$ con la JD; $r=-0.50 ; p<0.001$ con la JP; $\mathrm{y}, r=-0.47$; $p<0.001$ con la JI), lo que puede presagiar un efecto moderador en la relación básica aludida. Al mismo tiempo, en línea con estudios previos de la literatura, dichas variables de justicia no muestran un efecto directo importante sobre la ciberpereza lo que confirma nuevamente el aserto doctrinal que propugna la conveniencia de interponer variables intermedias o moderadoras -como es el caso de nuestra investigación- para modelizar adecuadamente el contexto organizacional concreto que estudiamos. También habría que hacer notar la influencia nula de la anomia en la ciberpereza ( $r=-0.07$; $p$ n.s.) y, sobre todo, la relación positiva de la JD con la ciberpereza $(r=0.17 ; p<0.05)$ cuando actúa directamente, lo que parece apoyar aún más las tensiones previstas de la relación básica mencionada si no es acomodada por nuevas variables explicativas como, en nuestro caso, podría ser la anomia. De cualquier modo, son los efectos de interacción entre las variables los que constituyen el interés primordial de nuestro trabajo.
Para contrastar las hipótesis se llevó a cabo un análisis de regresión múltiple jerárquica (Cohen y Cohen, 1983) en la que la ciberpereza se introdujo como variable dependiente. Antes, dado que las variables de justicia y la anomia afectan en sentido inverso a la ciberpereza, el continuo anomia-'ausencia de anomia' fue invertido. Por tanto, es de esperar que la nueva variable, la 'ausencia de anomia', y las justicias, interactúen entre ellas en la misma dirección con la ciberpereza. A continuación, el análisis se llevó a cabo en tres pasos: en el primero, paso 1, se introdujeron las variables de control, en el paso 2 todas las variables independientes y la anomia. En el paso 3, como establece el método referenciado, las interacciones de anomia con cada una de los tres tipos de justicia. Las variables fueron tipificadas consiguiéndose niveles aceptables de mulicolinearidad (Aiken y West, 1991). Los resultados, junto a los coeficientes Beta estandarizados, se exponen en la Tabla 3. Dicha tabla muestra el cambio en el $R^{2}$ en cada paso de las regresiones, así como la significación de cada Beta y del modelo final con todas las variables.

Las interacciones de la anomia con cada una de las tres justicias (Ausencia de anomia x JD; Ausencia de anomia x JP; y Ausencia de anomia x JI) fueron utilizadas para examinar las hipótesis (Paso 3; Tabla 3). La Tabla 3 muestra que las interacciones-introducidas conjuntamente-explican un incremento significativo en $R^{2},\left(\Delta R^{2}=0.065 ; p<0.001\right)$.

Además, un análisis individual a las tres interacciones (Ausencia de anomia x JD; Ausencia de anomia X JP; y Ausencia de anomia x JI) nos revela la existencia de un efecto moderador significativo de la anomia, operacionalizada como 'ausencia de anomia', sobre la relación entre la JD $(B=-0.281 ; p<0.05)$, la JP $(B=$ $-0.430 ; p<0.01)$ y la JI $(B=-0.217 ; p<0.05)$ y la ciberpereza. Ello indica que entre los profesores menos anómicos, comparados con los más anómicos, sus percepciones de justicia (en cualquiera de sus tres variantes) se relacionan más negativa e intensamente con su ciberpereza, haciéndoles, por tanto, disminuir su uso indebido de los servicios de Internet de su universidad. Por ello las tres hipótesis propuestas encuentran apoyo $\mathrm{y}$, por tanto, son aceptadas.

Tabla 2. Medias, desviaciones estándar, correlaciones y fiabilidades

\begin{tabular}{lccccccccc}
\hline Variables & M & SD & 1 & 2 & 3 & 4 & 5 & 6 & 7 \\
\hline 1. Sexo & 1.36 & 0.48 & ---- & & & & & \\
2. Edad & 2.78 & 0.88 & -0.08 & --- & & & & & \\
3. Anomia & 3.57 & 1.29 & $0.17^{* *}$ & 0.01 & $(0.839)$ & & & \\
4. Justicia Distributiva (JD) & 3.54 & 1.52 & -0.10 & 0.01 & $-0.48^{* * *}$ & $(0.921)$ & & \\
5. Justicia Procedimental & 3.05 & 1.43 & -0.04 & $-0.12^{*}$ & $-0.50^{* * *}$ & $0.59^{* * *}$ & $(0.961)$ & & \\
6. Justicia Interpersonal (JI) & 4.34 & 1.74 & -0.12 & -0.04 & $-0.47^{* * *}$ & $0.43^{* * *}$ & $0.47^{* * *}$ & $(0.949)$ & \\
7. Ciberpereza & 2.87 & 1.57 & $-0.25^{* * *}$ & $-0.14^{*}$ & -0.07 & $0.17^{*}$ & 0.06 & 0.12 & $(0.862)$ \\
\hline
\end{tabular}

Nota: Los números en cursiva, y entre paréntesis, en la diagonal son coeficientes alfa. Sexo: $1=$ hombre, $2=$ mujer; Edad: $1=23$ a $30 ; 2=31$ a $40 ; 3=41$ a $50 ; 4=51$ a $60 ; 5=61$ a 70; $6=$ 71 a 75 . Niveles de significación: $* \mathrm{p}<0.05 ; * * \mathrm{p}<0.01 ; * * * \mathrm{p}<0.001$. 
Tabla 3. Resultados del Análisis de Regresión Múltiple Jerárquico entre las variables Justicia Distributiva, Justicia Procedimental, Justicia Interpersonal, Anomia y Ciberpereza

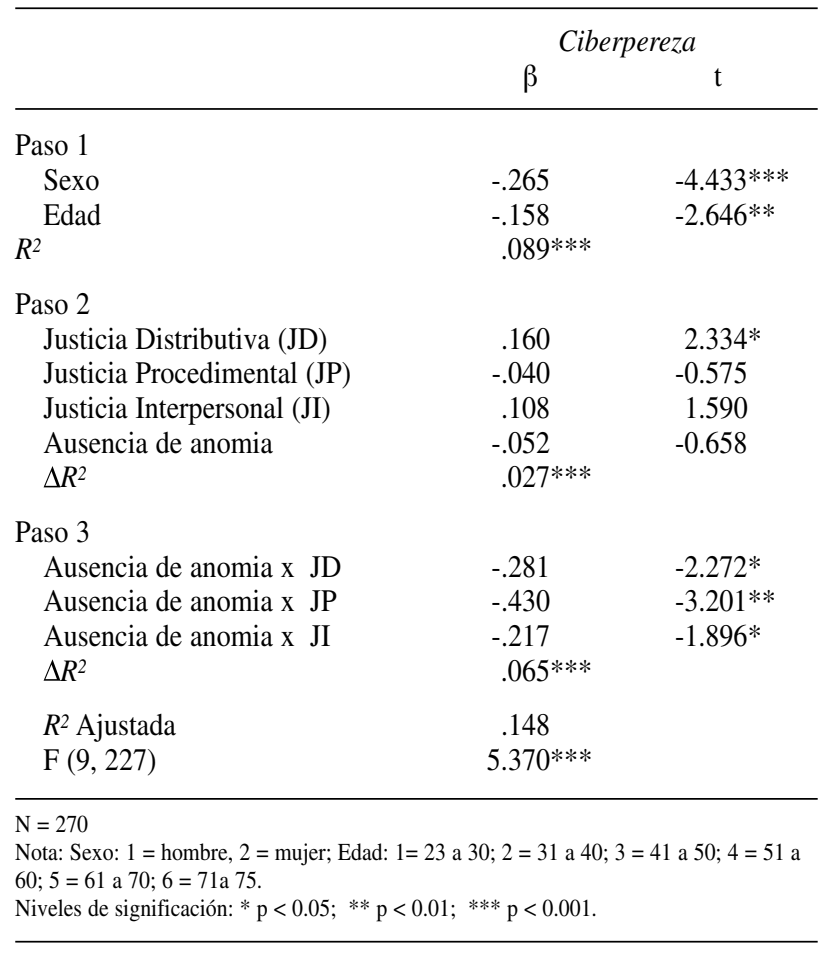

\section{Discusión}

El objetivo de esta investigación fue suministrar apoyo a la tesis de que la anomia modera la relación tradicionalmente aceptada entre percepciones de justicia y la conducta organizativa y, más concretamente, en nuestro caso, la conducta organizativa desviada en el uso de Internet o ciberpereza. El apoyo empírico obtenido debe permitirle a la dirección de recursos humanos abrir su abanico de opciones a la hora de combatir la ciberpereza. Consecuentemente, una nueva perspectiva de mayor éxito en la lucha contra tal conducta desviada debiera, a la luz de las estadísticas sobre las pérdidas que genera, mejorar significativamente el rendimiento organizacional. Tales asertos, en consonancia con los objetivos básicos del estudio, exigen una exhaustiva reflexión sobre las conclusiones e implicaciones más importantes a las que contribuye la presente investigación.

En primer lugar, los coeficientes (relaciones) negativos del sexo con la ciberpereza $(B=-0.265$; $p<$ $0.001)$ del Paso 1 podrían explicarse a través de las teorías sobre "venganza y atribución de culpas". Hay estudios (Cash et al., 1977; Dobbins et al., 1983) que sostienen que las mujeres tienen en menor medida un "estilo de atribución sesgado", es decir, tienden menos que los varones a culpabilizar exageradamente a agen- tes externos por acontecimientos negativos y a atribuirse, como éxitos propios, los positivos. Como consecuencia, las mujeres tienden en menor medida que los varones, a "culpabilizar" a la organización y, en consecuencia, podrían mostrar menos represalias y ciberpereza. El coeficiente negativo de la edad con la ciberpereza $(B=-0.158 ; p<0.01)$ pudiera reflejar un uso menos frecuente de Internet entre los profesores de más edad. Así, Internet forma parte de una nueva tecnología a la que los profesores más veteranos acceden muy puntualmente en la universidad estudiada.

En segundo lugar, en consonancia con investigaciones previas, la justicia organizativa aparece, una vez más, como un constructo de poderosa influencia pero de difícil encauzamiento a la hora de integrarlo en modelos que expliquen el comportamiento organizacional. Parece reflejar valores culturales profundos de la organización toda vez que muestra necesitar de la mediación de aspectos más 'visibles', como podrían ser variables intermedias de ambiente y clima organizacional, para concretarse en conductas. Por ejemplo, vimos como la doctrina propone constructos como, la confianza, el apoyo organizacional, el estatus laboral, etc., para materializar los importantes efectos de la justicia organizativa en el comportamiento organizacional. El proceder de la justicia organizativa como reflejo de valores culturales y, por qué no, como un valor cultural en sí mismo, parece encontrar apoyo en los resultados de nuestra investigación. En efecto, la anomia, por definición, podríamos asociarla a un buen 'indicador' de falta de valores organizacionales, lo que es confirmado por la importantísima interacción negativa entre la anomia y dichas variables de justicia $(r=$ $-0.48 ; p<0.001$ con la JD, $r=-0.50 ; p<0.001$ con la JP; y, $r=-0.47$; $p<0.001$ con la JI). Ello parece indicar que la percepción de una organización injusta se constituye en una situación generadora de anomia, desde el momento en que 'el valor justicia' es el que la desencadena cuando la organización es percibida como injusta. La caída de dicho 'valor justicia' (muy importante por otra parte) puede muy bien explicar la interacción-moderación-demostrada en nuestro estudio.

En tercer lugar, parece que las percepciones de injusticia pueden no tener un significado homogéneo para todos los miembros de la organización. La interpretación de una situación de justicia no es por tanto única, ni absoluta, entre los empleados. Habría que cifrar la propia situación personal y organizativa que vive cada sujeto que tilda a su empresa de injusta con él. En el presente estudio, esa situación viene determinada por la anomia. En efecto, los profesores más anómicos interpretaron la justicia organizativa en dicha clave y, consecuentemente, mostraron más ciberpereza, comparados con la generalidad de la población estudiada. Por contra, las distintas formas de justicia se mostraron, sin la moderación de la anomia, como un contexto neutro, e incluso contradictorio en el caso de la JD ya que presenta una vinculación positiva con el 
ciberpereza $(B=0.160 ; p<0.05$; Tabla 3, Paso 2). Este es un ejemplo de la tradicional variable supresora "neta" donde JD está negativamente correlacionada con ciberpereza ( $r=-0.48 ; p<0.001)$, pero tiene un coeficiente de regresión positivo $(B=0.160 ; p<0.05)$. La causa suele estar en que JD suprime la diferencia de error en JP y/o JI, en lugar de mostrar la varianza "adecuada" sobre la ciberpereza (Cohen y Cohen, 1983). Sin embargo, su influencia, dentro el contexto de la anomia, le devuelve su potencial explicativo sobre la conducta organizativa-la ciberpereza, en nuestro caso-que la literatura de comportamiento organizacional, con amplio consenso, previamente le asignaba. En efecto, moderada por la anomia, podemos ver como la JD pasa de una relación positiva $(B=0.160 ; p<$ 0.05 ; Tabla 3 ; Paso 2$)$ a negativa $(B=-0.281 ; p<0.05$; Tabla 3; Paso 3), que probablemente se mostraría aún más intensa puesto que se introduce en el paso 3, es decir, cuando todas las demás variables ya han 'capitalizado' su efecto sobre la ciberpereza.

Por último, llama la atención la JP, que a pesar de su teórica inferioridad con respecto a la JD, ya que ésta es admitida como la más influyente por su gran potencial en satisfacción de necesidades, haya mostrado un efecto moderador superior $(B=-0.430 ; p<0.01)$ que la mencionada JD $(B=-0.281 ; p<0.05)$. Quizá la razón de tal circunstancia la podríamos encontrar en la naturaleza de la ciberpereza. Esta conducta desviada y en Internet, por definición, están estrechamente incardinadas en los procedimientos y rutinas de trabajo del profesorado investigador. Probablemente Internet sea una de las herramientas de trabajo más importante de un profesor universitario. Ante una situación de injusticia organizativa la literatura sobre comportamiento organizacional suele, si bien de forma aún tímida, ser selectiva sobre qué 'tipo de respuesta' puede esperase de cada forma de injusticia. En nuestro caso, pensamos que los datos obtenidos pueden dar apoyo a la tesis de que la JP, por su definida asociación a los procedimientos de trabajo del profesorado y, por tanto, con la práctica diaria del uso de Internet, puede constituir a éste último en 'chivo expiatorio' a través de la ciberpereza. Es decir, la injusticia procedimental (JP) percibida puede apuntar a Internet como 'blanco de rebeldía' contra el que el profesorado reacciona. Ello explicaría la primacía de la JP en influencia moderadora, con respecto a la JD y a la JI, las cuales se muestran claramente más débiles, en especial la JI $(B=-0.217 ; p<0.05)$.

\section{Implicaciones, limitaciones y recomendaciones}

El presente estudio tiene importantes implicaciones, tanto prácticas como teóricas, para el desarrollo de posibles estrategias de control de la ciberpereza en las organizaciones. En alusión a las prácticas, la incorporación de la anomia además de su relación con los constructos clásicos de justicia organizativa, parece exigir a las organizaciones el fomento de la existencia de una normativa firme, clara y justa, que impida la aparición de escepticismos y actitudes amorales, mediante el consenso de un entramado normativo que genere certidumbre, coherencia y estabilidad. En tal propósito parece importante un apoyo oficial a dicha normativa, legitimando las reglas de régimen interno de la universidad mediante su elaboración participada y recogiendo el sentir de toda la jerarquía e, incluso, de los empleados docentes. Ello capitalizaría la que parece ineludible necesidad de fomentar el soporte normativo y la transmisión de valores, sobre todo en el puesto de trabajo, para evitar desarraigos anómicos que parecen perjudicar el uso saludable de Internet y, por ende, la calidad de la institución pública universitaria. Tal forma de proceder, permitiría, a nuestro juicio, una unidad de criterio que parece entroncar, claramente, con las conclusiones vistas en la investigación.

El éxito del modelo propuesto que incorpora la anomia como moderador de una situación de justicia organizativa en el control de la ciberpereza, nos puede llevar a pensar que el éxito de un moderno sistema de control de conducta desviada, y de la ciberpereza en particular, debe apoyar los planteamientos 'situacionales' que rechacen la creencia de que los comportamientos indisciplinados son producto de atributos más o menos permanentes en los empleados, como la pereza, o la irresponsabilidad. Por tanto, parece conveniente la apuesta por posturas que señalen la etiología de la ciberpereza como una consecuencia de un ambiente organizacional que adolece de valores de justicia, certidumbre, orden y claridad ético-normativa.

En esta línea, la dirección de recursos humanos de las organizaciones quizá deba incluir en sus periódicos estudios de clima, para calibrar niveles de satisfacción, motivación y percepciones justicia, una auditoría de la salud 'eunómica' de su organización a fin de intentar reducir la ciberpereza. En esta línea debemos explicitar nuestra apuesta por el aprendizaje organizacional, como herramienta capaz de integrar el 'conocimiento' y conseguir esas mejoras conductuales en los miembros de la organización. En efecto, el conocimiento es algo más que un mero dato o un flujo informativo cargado de datos (Davenport y Prusak, 1999): es un marco de experiencias, valores (ijusticia?), información y 'saber hacer' (¿normas?) del individuo que permite la acumulación de nuevo conocimiento y la acción eficaz. El aprendizaje organizacional nos permite integrar el conocimiento para conseguir esas mejoras conductuales en los miembros de la organización. Requiere herramientas, por tanto, que permitan convertir el conocimiento individual en colectivo. En esta línea, Senge (1990) sientó las bases de las denominadas 'organizaciones inteligentes.' El aprendizaje organizacional acoge el déficit de un uso adecuado de Internet-ciberpereza-como un 'problema' no estructurado que requiere la aplicación de un cambio en las estructuras mentales de los sujetos implicados. 
El control de la ciberpereza, por tanto, creemos requiere cambios profundos en las estructuras mentales de sus protagonistas ya que partimos de la hipótesis de que la naturaleza de la ciberpereza puede tener un fuerte matiz anómico que nos enfrenta a un problema profundamente desestructurado. Ya Senge (1990) o Rouse et al. (1992), entre otros, hacen referencia a la capacidad del aprendizaje organizacional de movilizar el modelo mental o ese conjunto de imágenes internas que el sujeto posee del mundo organizacional que le rodea, que condicionan su visión de éste y que influyen poderosamente en su comportamiento y aprendizaje. En suma, un 'saber aprender' más allá del 'saber cómo se hace', know-how, o 'saber cómo funciona', knowwhy.

En lo que a posibles implicaciones académicas se refiere, parece conveniente resaltar la incorporación de la anomia al escenario de la literatura sobre ciberpere$\mathrm{za}$, que en lo que a su influencia moderadora conducida en nuestro estudio se refiere, se muestra inédita. También parece que el uso replicado de estas variables fortalece la apuesta metodológica de influencia de las relaciones de intercambio social en los comportamientos desviados, y que la anomia parece mostrarse como un nuevo regulador de dichos intercambios sociales.

Difícilmente ubicable en la literatura sobre la motivación en las organizaciones, los resultados de esta investigación sugieren que el estudio de la anomia en las organizaciones debería abrirse paso en la teoría a la luz del éxito que, en la modesta presente investigación, ha presentado y que destacamos como otra implicación doctrinal. En efecto, la anomia, un fenómeno social que califica y explica, en buena parte, la evolución y desarrollo de nuestras sociedades durante el último siglo, creemos que no ha gozado del exigible paralelismo pertinente, en el estudio del comportamiento humano en las organizaciones. Quizá tendríamos que empezar a plantearnos si el problema de la eficacia en el desempeño de nuestros recursos humanos, con sus conductas desviadas concomitantes — como la ciberpereza-nos exigiría ahora no sólo hablar de 'empleados desmotivados' sino, también, de 'empleados anómicos.'

Por último, convendría advertir de ciertas limitaciones que creemos adolece nuestra investigación. En primer lugar, la institución académica investigada es una universidad de relativa reciente creación, y con una plantilla joven y con menor nivel de funcionarios que otras consolidadas en el territorio nacional e internacional. Tal circunstancia puede poner límites a la extrapolación de las conclusiones de la presente investigación a otras universidades y organizaciones. En segundo lugar, el colectivo del profesorado universitario reúne ciertas condiciones de trabajo inherentes, muchas veces, a las peculiaridades de las tareas docentes e investigadoras-flexibilidad horaria, control por objetivos, menor supervisión, entre otras-que también impedirían extrapolaciones a otros colectivos de trabajadores, tanto públicos como privados. Por último, el estudio ha seguido un método transversal (cross-sectional) lo que implica que todos los datos tienen en los profesores la misma fuente (auto-cumplimentados) y un único registro temporal. El riesgo de una capitalización $\mathrm{R}$ cuadrado debida al problema de la varianza del método común puede limitar también la generalización de nuestros resultados.

En conclusión, dada su probada interacción con las percepciones de justicia organizativa en general y con la justicia procedimental (JP) en particular, la presente investigación subraya la utilidad de diagnosticar patologías organizativas de tipo anómico para predecir el uso indebido de Internet en el centro de trabajo. A la luz de tales resultados, en los programas empresariales de estudio de clima y cultura organizativa parece aconsejable incorporar como nueva variable de estudio la anomia en las organizaciones.

\section{Extended Summary}

Anomia (from the Greek, an-: absence, and -nomos: law) describes pessimistic feelings such as social detachment and little faith in human relations. This study examines the moderating role of anomia in the relationship between employees' perceptions of organizational justice and workplace Internet deviance (or Cyberloafing). The proposed model suggests that anomia interacts with organizational justice and cyberloafing by tightening their theoretical negative association.

While the mass introduction of Internet into organizations over the last decade has undoubtedly served to strengthen and catalyze their production processes (e.g., e-service, virtual groups, teleworking), it has also opened the door to a new type of workplace deviance: employees surfing the Internet instead of working. This particular new form of negative deviance in the workplace has been labeled 'cyberloafing' (Kamins, 1995) and could be classified as a DWB targeting the organization, since the cyberloafer acts directly against the company's Internet system (Bennett \& Robinson, 2000; Lim, 2002; Robinson \& Bennett, 1995). Lim defines Internet misuse as “... any voluntary act of employees' using their companies' Internet access during office hours to surf non-job related Web sites for personal purposes and to check (including receiving and sending) personal e-mail (2002:677)." 
Cyberloafing has become a widespread and costly problem for organizations. A survey of 1,000 workers in the US revealed that $64 \%$ of them surf the Internet for personal purposes during working hours (The Straits Times, 2000). Cyberloafing is responsible for a $30-40 \%$ drop in employee productivity (Verton, 2000). With things as they are, corporations are striving for stricter regulation of internet use, whereas management research urgently seeks its antecedents in order to understand better the occurrence of cyberloafing. Previous research offers a wide range of potential and empirically supported antecedents of cyberloafing. Organizational injustice is a frequently cited cause of misconduct. However, the direct relationship between the equity theory and employee outcomes -and workplace deviance in particular- provides relative deprivation models that only provide a partial explanation (see, e.g., Aquino et al., 1999; Aryee et al., 2002). This equity scenario seems to require further variables to represent the mechanism through which organizational justice is capable to predict employees' workplace deviance and cyberloafing in particular.

Hypotheses - In contrast to Durkeim and Merton, who were concerned with the cultural determinants of anomie, Srole (1956) sought to describe specific traits of an anomic individual. According Srole, each one of his five-item scale represents one distinct facet of anomia (1956:712-713): (1) the individuals' sense that leaders are indifferent to their needs; (2) their perception of the social order as essentially fickle and unpredictable; (3) the view that they, and people like them, are retrogressing; (4) the sense of life meaningless, and (5) their perception that their immediate personal relationships are no longer predictive or supportive. As we see later, we expand the one-dimensional Srole's five-item scale with three added items strengthening the assessment of employees' sense of work (or organizational) anomia.

If an individual's sense of identity is not fulfilled by membership in the organization, or if she senses that the organization does not value her, she will view her work merely as a small part of who she is. When injustice prevails within organizations, no single set of values for employees and management is followed or adhered to. Confusion results and it can cause employees to become disoriented and isolated. Therefore, anomia may interact with justice-cyberloafing link because injustice perceptions lead employees to a sense to be unvalued by their organization, and thus to a generalized skepticism and estrangement of their organization, which making them more prone to getting upset and more emotionally willing to engage cyberloafing. Hypotheses: The perceptions of Distributive Justice (1a) and/or Procedural Justice (1b) and/or Interactional Justice (1c) will be more strongly negatively associated with cyberloafing for employees with comparatively lower sense of anomia.

\section{Method}

Participants: Data were collected from 270 $(17.46 \%)$ of the 1,547 teachers at a Spanish public university by Intranet e-mail. The sample was comprised of $63.6 \%$ males and $35.4 \%$ females and, while $40 \%$ were 40 years old or younger, only $4.2 \%$ were older than 60 . Most $(68 \%)$ of the sample held tenured positions while the remainder did not. Contextual conditions that lead us to presume varying willingness to respond were not found. The research project received prior official approval and IP addresses were unidentifiable and the surveyors were so informed so as to avoid their reticence and interference in responses. Additionally, the sample descriptive structure is quite similar to the population data. Effectively, in the universe $932(60.28 \%)$ of the teachers held tenures, while the remaining 624 had some other type of contract. By age, $501(32.5 \%)$ were 40 years old or younger and only $89(5.7 \%)$ were 60 or above. By gender, $68 \%$ were male and $32 \%$ female.

\section{Results}

Multiple Hierarchical Regression results support that anomia acts as a moderator of the justice-cyberloafing link because the perceptions of the three types of organizational justice (distributive, procedural, and interactional) among employees with low, as compared to high anomia, have a stronger negative relationship with cyberloafing. This anomia's moderation effect was especially intense in the case of procedural justice.

\section{Main Conclusions}

The findings contribute to a better understanding of the conditions under which organizational justice perceptions are capable to affect cyberloafing. Anomia is supported as one of those conditions and hence, the control of anomic feelings may allow organizations to stifle cyberloafing. Other implications of the results for control of cyberloafing and monitoring the anomia are discussed, and future research directions are offered.

Research limitations/implications - The researched employees have job conditions inherent to the peculiarities of the public sector that may limit the ability to extrapolate the findings in the private sector. Furthermore, the study could suffer from monomethod/source bias. The performance of the constructs used in the present research, as well as their implications, therefore, could vary. 


\section{References}

Adams, J.S. (1965). Inequity in social exchange. In Advances in Experimental Social Psychology, Berkowitz, L., ed. Academic Press, New York, USA.

Aiken, L.S. y West, S.G. (1991). Multiple regression: Testing and interpreting interactions. Newbury Park CA: Sage.

Allport, F.H. (1924) Social Psychology. Boston, MA: Houghton Mifflin.

Analoui, F., y Kakabadse, A. (1991) Sabotage. London: Mercury.

Aquino, K., Galperin, B.L., y Bennett, R. (2004) Social Status and Aggressiveness as Moderators of the Relationship Between Interactional Justice and Workplace Deviance. Journal of Applied Psychology, 34, 5, 1001-1029.

Aquino, K., Lewis, M. U, y Bradfield, M. (1999). Justice constructs, negative affectivity, and employee deviance: A proposed model and empirical test. Journal of Organization Behavior, 20, 1073-1091.

Aryee, S., Budhwar, P.S., y Chen, Z.X. (2002). Trust as a mediator of the relationship between organizational justice and work outcomes: test of a social exchange model. Journal of Organizational Behavior, 23, 267-285.

Bass, B. (1990) Bass \& Stogdill's Handbook of Leadership: Theory, Research, and Managerial Applications, New York, NY: Free Press.

Blau, P. M. (1964). Exchange and Power in Social Life. John Wiley \& Sons.

Bollen, K. A. (1989). Structural Equations with Latent Variables. New York: John Wiley \& Sons.

Bronikowski, L. (2000). Esniff.com sniffs out cyberslacking. ColoradoBiz, 27 (11), 46.

Cash, T. F., Gillen, B., y Burns, D. S. (1977). Sexism and beautyism in personnel consultant decision making. Journal of Applied Psychology, 62, 301-310.

Cohen, D. (1995) Ethics and Crime in Business Firms: Organizational Culture and the Impact of Anomie, In Freda Adler and William Laufer, (Ed.), The Legacy of Anomie Theory, New Brunswick: Transaction Publishers, 183-206.

Cohen, J. y Cohen, P. (1983) Applied multiple regression/correlation analysis for the behavioral sciences (2nd ed.). Hillsdale, NJ: Erlbaum.

Davenport, T.H. y Prusak, L. (1998), Working Knowledge. How organisations manage what they know. Harvard Business School Press.

DiBattista, R.A. (1989). Designing a program to manage the risk of sabotage. Supervision, 6-8.

DiBattista, R.A., (1996). Forecasting Sabotage Events in the Workplace. Public Personnel Management, 25, 41-52.

Dobbins, G., Pence, E., Orban, J., y Sgro, J. (1983). The effects of sex of the leader and sex of the subordinate on the use of organizational control policy. Organizational Behavior and Human Performance, 32, 325-343.

Durkheim, E. [1893 (1973)], De la Division du Travail Social. Paris, PUF.
Durkheim, E. [1897 (1973)] Le Suicide. Paris, PUF.

EcoDiario. (2008). EcoDiario April 23, 2008. http://ecodiario.eleconomista.es/espana/noticias/ 496073/04/08/ Chacon-deja-a-los-militares-sin-As-Marca-e-Interviu-enInternet.html

Folger, R., y Cropanzano, R. (1998) Organizational justice and human resource management. Thousand Oaks, CA: Sage Publications.

Greenberg, J. (1990) Employee theft as a reaction to underpayment inequity: The hidden cost of pay cuts. Journal of Applied Psychology, 75, 561-568.

Hackman, J.R., y Oldham, G.R. (1976) Motivation through the design of work: Test of a theory. Organizational Behavior and Human Performance, 15, 250-279.

Hagedorn, R., y Labovitz S. (1968) A Reply to Gibbs and Martin, and Tennant, Social Problems.

Hodson, R. (1999) Organizational anomie and worker consent. Work and Occupations, 26, 292-323.

Homans, G. (1961) Social Behavior: Its Elementary Forms. New York: Harcourt Brace.

James, L. R., Mulaik, S. A., y Brett J. M. (1982) Causal analysis: Assumptions, models, and data. Beverly Hills: Sage

Jöreskog, K.G. y Sörbom, D. (1979). In Advances in Factor Analysis and Structural Equation Models. Magidson, J. ed., Cambridge, Massachusetts: Abt Books.

Kamins, A. (1995). Cyber-loafing: Does employee time online add up to net losses? New York Daily News, July.

Lim, V.K.G. (2002). The IT way of loafing on the job: cyberloafing, neutralizing and organizational justice. Journal of Organizational Behavior, 23, 675-694.

Long, J. S. (1983) Covariance structure models: An introduction to LISREL; Paper Series on Quantitative Application in the Social Science. London: Sage Publications, 34.

MacIver, R.M. (1950). The Ramparts We Guard. Macmillan, New York.

Management Issues News. (2004). Does cyber-loafing undermining productivity? Management Issues News, 21 July, News \& Research. From http:/www.mangementissues.com/dsiplay page.asp?section=research\&id=141.

Marks, S. R. (1974), Durkheim's Theory of Anomie. American Journal of Sociology, 80, 329-63.

McClosky, H. y Schaar, J.H. (1965), Psychological Dimensions of Anomy. American Sociological Review, 32, 14-27.

Menard, S. (1995) A developmental test of Mertonian anomie theory. Journal of Research in Crime and Delinquency, 32, 136-174.

Merton, R.K. (1957) Priorities in scientific discovery, American Sociological Review, 22, 635-659.

Miller, C.R., y Buttler, E.W. (1966) Anomia and Eunomia: A Methodological Evaluation on Srole's Anomia Scale. American Sociological Review, 31, 400-406.

Moorman, R. H. (1991). The relationship between organizational justice and organizational citizenship behavior: Do fairness perceptions influence employee citizenship? Journal of Applied Psychology, 76, 845-855. 
Moorman, R. H., Blakely, G. L., y Niehoff, B. P. (1998). Does organizational support mediate the relationship between procedural justice and organizational citizenship behavior? Academy of Management Journal, 41, 351357.

Neuman, J.H., \& Baron, R.A. (1998). Workplace violence and workplace aggression: evidence concerning specific forms, potential causes, and preferred targets. Journal of Management, 24, 391-419.

Niehoff, B.P. y Moorman, R.H. (1993) Justice as a mediator of the relationship between methods of monitoring and organizational citizenship behavior, Academy of Management Journal, 36, 527-556.

Potter, R.H. (1989) Ethics, discipline, and human nature: a new look al management and deviance, Industrial Management, 31, 2, 14-21.

Robinson, S.L., y Bennett, R. J. (1995). A typology of deviant workplace behaviors: A multidimensional scaling study. Academy of Management Journal, 38, 555-572.

Robinson, S.L., y Bennett, R.J. (1997). Workplace Deviance: Its definition, its manifestations, and its causes. In Lewicki, R. J. Bies, \& B. H. Sheppard (Eds.), Research on negotiation in organizations (pp. 3-27). Greenwich, CT: Jai Press Inc.

Robinson, S.L., y Kraatz, M. S. (1998). Constructing the reality of normative behavior: the use of neutralization strategies by organizational deviants. In R. Griffin, A. O'Leary-Kelly, y J. Collins (Eds.), Dysfunctional behavior in organizations: Violent \& deviant behavior (pp. 203-220). Part A. Stamford, CT: JAI Press.

Rouse, W.B., Cannon-Bowers, J.S., \& Salas, E. (1992) The role of mental models in team performance in complex systems, IEEE Transactions on Systems, Man, and Cybernetics, 22, 1296-1307.

Senge, P. (1990), The Fifth Discipline, Doubleday Plub., New York
Shedd, J. y Bachrach, S. (1991) Tangled hierarchies, San Francisco, CA: Jossey-Bass.

Sieh, E.W. (1987). Garment workers: perceptions of inequity and employee theft. British Journal of Criminology, 27, 174-191.

Skarlicki, D. P., Folger, R., y Tesluk, P. (1999) Personality as a moderator in the relationship between fairness and retaliation. Academy of Management Journal, 42, 100-108.

Skarlicki, D.P., y Folger, R. (1997) Retaliation in the workplace: The roles of distributive, procedural, and interactional justice. Journal of Applied Psychology.

Srole L. (1956) Social integration and certain and certain corollaries: an exploratory study, American Social Review, 21, 709-716.

Sykes, G. M., y Matza, D. (1957). Techniques of neutralization: a theory of delinquency. American Sociological Review, 22, 664-670.

Taylor, G.S., y Zimmerer, T.W. (1992) Voluntary turnover among middle-level managers: An analysis of perceived causes, Journal of Managerial Issues, 4, 424-437.

The Straits Times. (2000). Cyberslackers at work. The Straits Times, 28 April, 4.

Thibaut, J. W. y Kelley, H. H. (1959). The social psychology of groups. New York.

Tucker J. (1993). Everyday forms of employee resistance: how temporary workers handle conflict with their employers. Soc. Forum, 8, 25-45.

Verton, D. (2000). Employers ok with e-surfing. Computerworld, 34, 1, 16.

Zellars, K.L., Tepper, B.J. y Duffy, M.K. (2002) Abusive supervision and subordinates' organizational citizenship behavior, Journal of Applied Psychology, 87, 1068-76.

Zoghbi, P. (2006). Fear in organizations: Does intimidation by formal punishment mediate the relationship between interactional justice and workplace internet deviance? Journal of Managerial Psychology, 21, 580-592.

Manuscrito Recibido: 12/12/2008

Revisión Recibida: 06/05/2009

Aceptado: 16/06/2009 
Anexo. Estructura completa del Análisis Factorial Confirmatorio (AFC) de todas las variables de este estudio

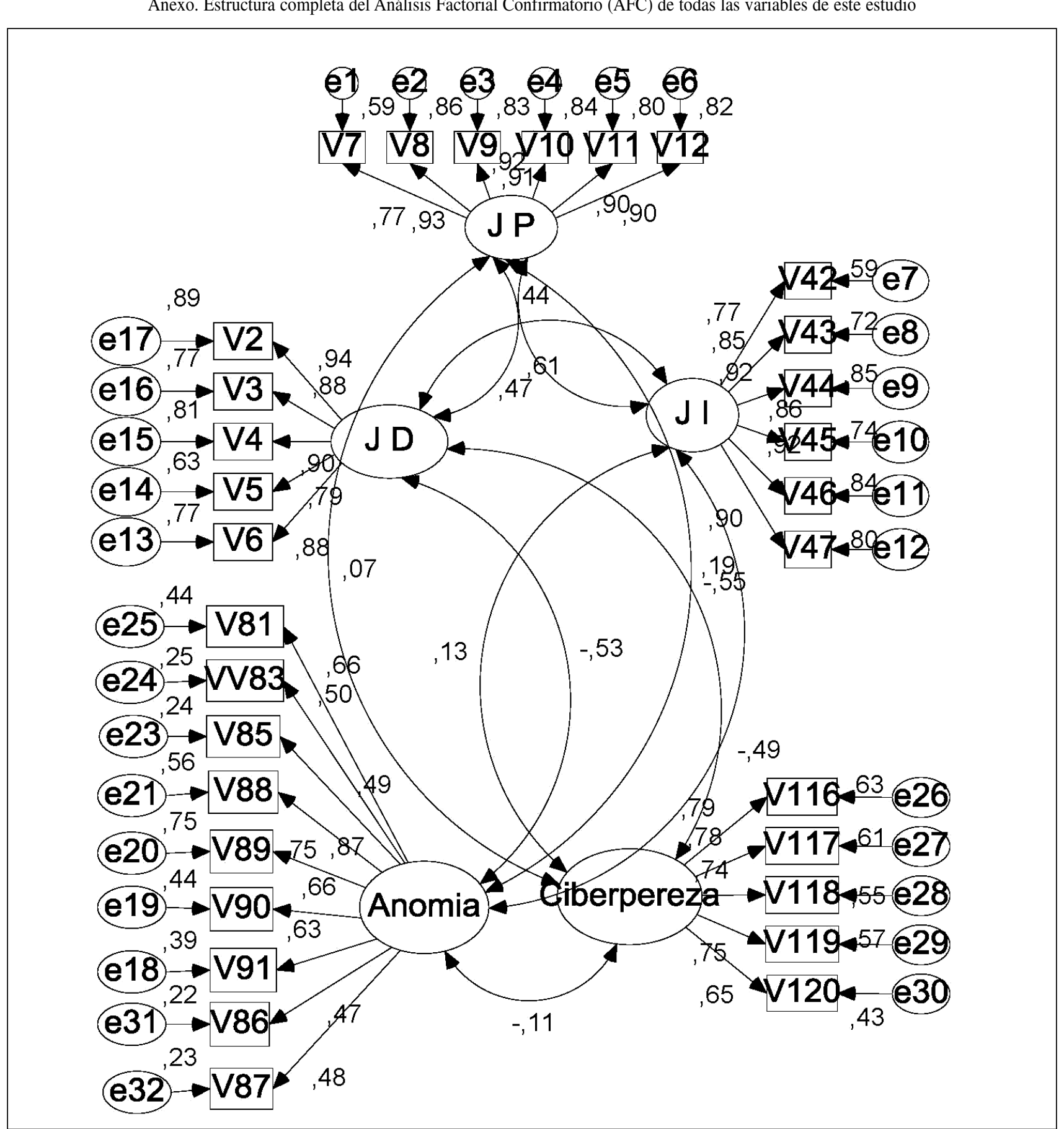

